\title{
Parathyroid adenoma causing spontaneous cervical haematoma and vocal cord palsy
}

R Ologunde, S Goh, S Ahmed, I Ahmad

Birmingham Heartlands Hospital, Bordesley Green East, Birmingham, B9 5SS, UK

USS Parathyroid

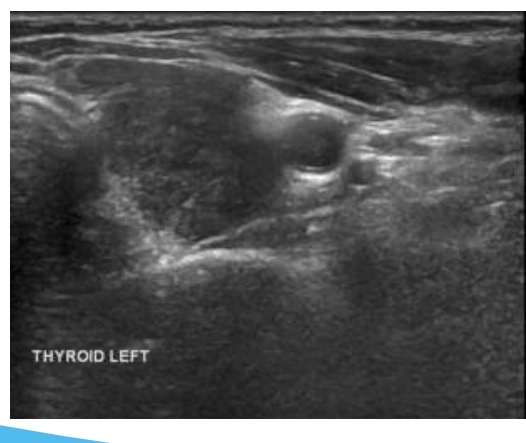

Sestamibi scan

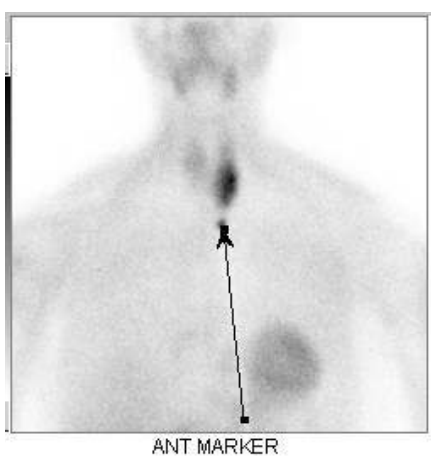

\section{Introduction}

Diffuse cervical-mediastinal haematoma attributable to spontaneous rupture of a parathyroid adenoma is an uncommon complication, but unrecognised can have potentially serious sequelae including major haemorrhage, dyspnoea, and death. We present an interesting case of spontaneous rupture of a parathyroid adenoma causing cervical-mediastinal haemorrhage and recurrent laryngeal nerve compression.

\section{Case}

We report a case of a 78-year-old lady, with a known history of parathyroid adenoma, presenting with airway compromise.

Our patient had a diagnosis of primary parathyroidism. A large parathyroid adenoma was identified extending to the superior mediastinum, localised to the left, on sestamibi scans and ultrasound. Further CT-imaging was used to visualise the extent of the adenoma and it was found to be $2.6 \times 2.0 \times 4.5 \mathrm{~cm}$ (AP $\times$ Trans $\times$ CC). Her corrected calcium was $3.22 \mathrm{mmol} / \mathrm{L}$ and PTH $45 \mathrm{pmol} / \mathrm{L}$. The patient initially declined surgical excision and was being considered for cinacalcet treatment.

However she presented to the emergency department with sudden onset stridor and hoarse voice, and subsequently developed odynophagia and a left sided neck swelling associated with overlying ecchymosis. Initial blood tests revealed cCa 2.63 mmol/L (2.20 - 2.60) and PTH 23.0 $\mathrm{pmol} / \mathrm{L}(1.60-7.20)$. Flexible nasendoscopy revealed an immobile left vocal cord in the para-median position. Computed Tomography (CT) scanning revealed the parathyroid adenoma at the lower pole of left lobe of thyroid gland and associated haematoma extending to the superior mediastinum, along the course of left recurrent laryngeal nerve. The haematoma resulted from spontaneous rupture of the arterial branch to the parathyroid gland, and had a mass effect causing right tracheal deviation and narrowing and oesophageal compression. The patient underwent an emergency left parathyroidectomy, hemithyroidectomy and evacuation of haematoma. There was an ill-defined and infiltrative appearance of the parathyroid adenoma identified at the time of surgery. Histology revealed a benign adenoma. Thyroid tissue revealed a lymphocyctic thyroiditis. Post-operatively the patients' blood work revealed cCa 2.48 and PTH 3.50.
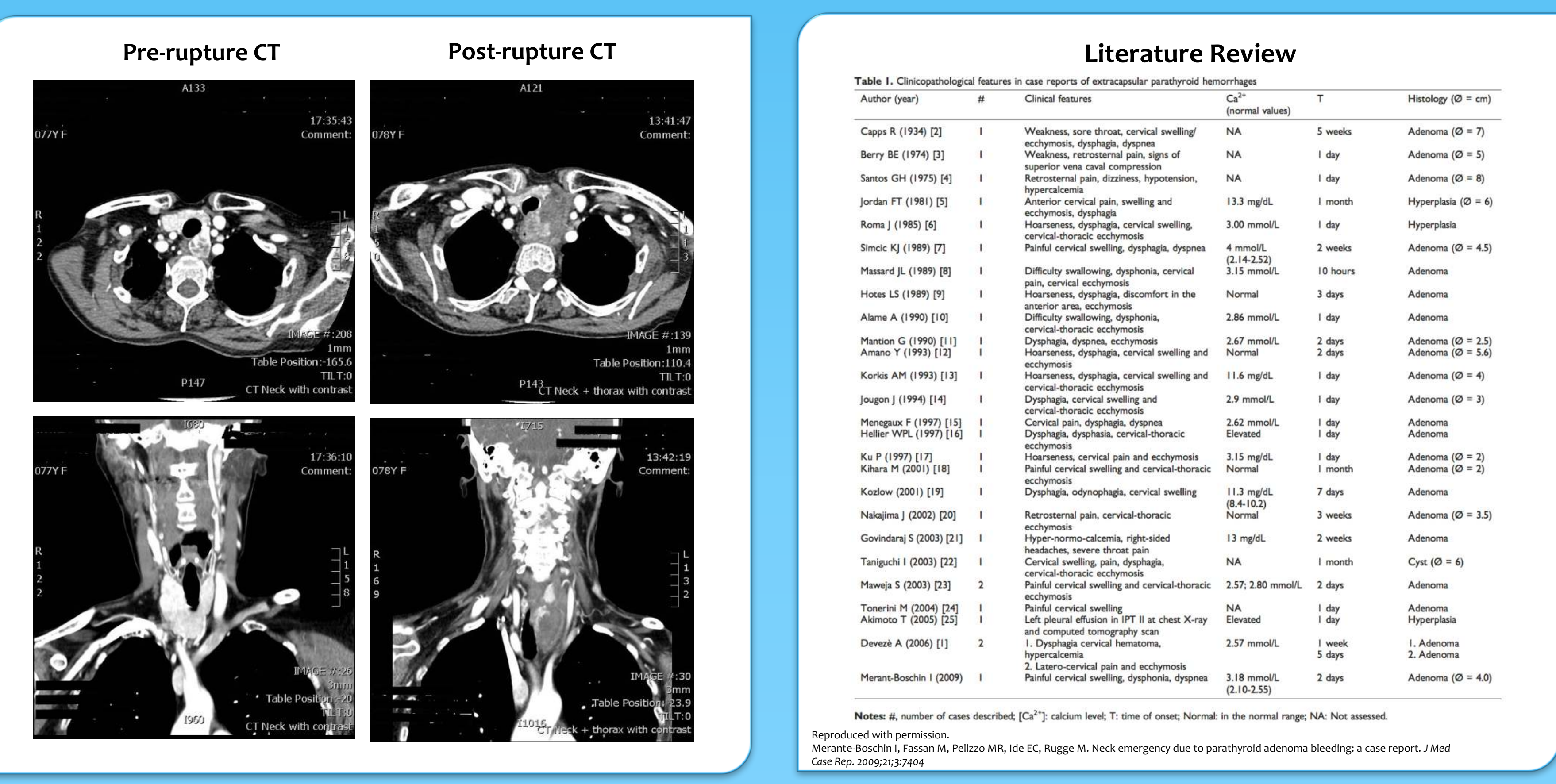

\section{Conclusion}

Spontaneous arterial rupture can occur in large parathyroid adenomas. Haematomas can lead to compression symptoms, vocal cord weakness and airway compromise. If the above "red flag" symptoms are present surgical exploration and excision are recommended. 